\title{
A Multi-Dimensional Flow Which Satisfies the Equation of Continuity
}

\author{
Dr. Hiteshwar Singh \\ Senior Professor (Rtd., Deptt. of \\ Mathematics, M.L.S.M. College \\ Darbhanga, India.
}

\author{
Ram Naresh Das \\ Univ. Deptt. of Mathematics, \\ L.N.M.U. Darbhanga, India.
}

\author{
Dhananjay Kumar Mishra \\ Univ. Deptt. of Mathematics, \\ L.N.M.U. Darbhanga, India.
}

\begin{abstract}
In this research paper, we have discussed upon a kind of generalized multi-dimensional incompressible flow. Let us suppose that at a distance $\left(\mathrm{x}_{1}, \mathrm{x}_{2}, \mathrm{x}_{3}, \ldots . \mathrm{x}_{\mathrm{n}}\right)$ from the origin, there be a flow. Then the generalized multi- dimensional means (n- dimensional) flow can be represented as,

$$
u_{1}=\frac{A x_{1}}{D^{n}}, \quad u_{2}=\frac{A x_{2}}{D^{n}}, \ldots \ldots, u_{n}=\frac{A x_{n}}{D^{n}}
$$

Here $\mathrm{D}$ is distance of the point $\left(\mathrm{x}_{1}, \mathrm{x}_{2}, \mathrm{x}_{3}, \ldots . \mathrm{x}_{\mathrm{n}}\right)$ from the origin and $\mathrm{A}$ is an arbitrary constant. This generalized multidimensional flow will satisfy the equation of continuity under incompressible condition.
\end{abstract}

Keywords: Equation of continuity, incompressible flow, ndimensional flow, generalized n-dimensional flow, distance of a point from the origin.

\section{INTRODUCTION}

As we know that the equation of continuity in fluid dynamics is also known as the equation of conservation of mass. Mathematically this equation will be presented as,

$$
\frac{\partial \rho}{\partial t}+\nabla(\rho \bar{q})=0
$$

Here $\rho$ is density of fluid and $\mathrm{q}$ is the fluid velocity at any point from the origin. But when we consider upon a nonhomogeneous incompressible fluid flow, then the rate of change of density $\frac{\partial \rho}{\partial t}$ will be zero. Now the equation of continuity will be written as

$$
\nabla(\rho \bar{q})=0 \Rightarrow \rho(\nabla \cdot \bar{q})=0
$$

or $\nabla \cdot \bar{q}=0$. If we the fluid velocity $\bar{q}$ is represented as, $\bar{q}=u \hat{i}+v \hat{j}+w \hat{k}$. Here $\mathrm{u}, \mathrm{v}, \mathrm{w}$ are components of velocity in $\mathrm{x}, \mathrm{y}, \mathrm{z}$ directions respectively. At this situation $\nabla \cdot \bar{q}=0$ will be presented as

$$
\frac{\partial(\vec{u} \cdot \hat{i})}{\partial x}+\frac{\partial(\vec{v} \cdot \hat{j})}{\partial y}+\frac{\partial(\vec{w} \cdot \hat{k})}{\partial z}=0
$$

or $\quad \frac{\partial u}{\partial x}+\frac{\partial v}{\partial y}+\frac{\partial w}{\partial z}=0$

\section{TEST OF FLUID FLOWS}

Now we choose a two dimensional flow as follow

$$
u(x, y)=\frac{A x}{x^{2}+y^{2}}, \quad v(x, y)=\frac{A y}{x^{2}+y^{2}}
$$

We put these fluid velocity components in $\frac{\partial u}{\partial x}+\frac{\partial v}{\partial y}=0$ to know whether these components satisfy the equation of continuity or not.

$$
\begin{aligned}
\frac{\partial u}{\partial x} & =\frac{\partial}{\partial x}\left(\frac{A x}{x^{2}+y^{2}}\right) \\
& =A \frac{\partial}{\partial x}\left(\frac{x}{x^{2}+y^{2}}\right) \\
& =\frac{A\left(x^{2}+y^{2}\right) \cdot \frac{\partial x}{\partial x}-A x \frac{\partial}{\partial x}\left(x^{2}+y^{2}\right)}{\left(x^{2}+y^{2}\right)^{2}} \\
& =\frac{A\left(x^{2}+y^{2}\right)-A x \cdot 2 x}{\left(x^{2}+y^{2}\right)} \\
& =\frac{A\left(x^{2}+y^{2}\right)-2 A x^{2}}{\left(x^{2}+y^{2}\right)^{2}} \\
& =\frac{A\left(y^{2}-x^{2}\right)}{\left(x^{2}+y^{2}\right)^{2}}
\end{aligned}
$$


Similarly, we will get, when we have $\frac{\partial v}{\partial y}$ as,

$$
\frac{\partial v}{\partial y}=\frac{\partial}{\partial y}\left(\frac{A y}{x^{2}+y^{2}}\right)=\frac{A\left(x^{2}-y^{2}\right)}{\left(x^{2}+y^{2}\right)^{2}}
$$

Now, we will check the equation of continuity of two dimensional flow,

$$
\begin{gathered}
\frac{\partial u}{\partial x}+\frac{\partial v}{\partial y}=\frac{A\left(y^{2}-x^{2}\right)}{\left(x^{2}+y^{2}\right)^{2}}+\frac{A\left(x^{2}-y^{2}\right)}{\left(x^{2}+y^{2}\right)^{2}} \\
=\frac{A\left(y^{2}-x^{2}+x^{2}-y^{2}\right)}{\left(x^{2}+y^{2}\right)^{2}}=0
\end{gathered}
$$

Thus the equation of continuity becomes satisfied here.

Now, we take a three dimensional flow, as follow

$$
\begin{aligned}
& u(x, y, z)=\frac{A x}{\left(x^{2}+y^{2}+z^{2}\right)^{3 / 2}} \\
& v(x, y, z)=\frac{A y}{\left(x^{2}+y^{2}+z^{2}\right)^{3 / 2}} \\
& w(x, y, z)=\frac{A z}{\left(x^{2}+y^{2}+z^{2}\right)^{3 / 2}}
\end{aligned}
$$

Again we will check whether these components of fluid velocity satisfies the equation of continuity or not. First of all we take $\mathrm{x}$-components, so

$$
\begin{aligned}
\frac{\partial u}{\partial x} & =\frac{\partial}{\partial x}\left(\frac{A x}{\left(x^{2}+y^{2}+z^{2}\right)^{3 / 2}}\right) \\
& =\frac{A\left(x^{2}+y^{2}+z^{2}\right)^{3 / 2} \frac{\partial x}{\partial x}-A x \cdot \frac{3}{2}\left(x^{2}+y^{2}+z^{2}\right)^{1 / 2} 2 x}{\left\{\left(x^{2}+y^{2}+z^{2}\right)^{3 / 2}\right\}^{2}}
\end{aligned}
$$

$$
\begin{aligned}
\frac{\partial u}{\partial x} & =\frac{A\left(x^{2}+y^{2}+z^{2}\right)^{3 / 2}-3 A x^{2}\left(x^{2}+y^{2}+z^{2}\right)^{1 / 2}}{\left(x^{2}+y^{2}+z^{2}\right)^{3}} \\
& =A\left\lfloor\frac{1}{\left(x^{2}+y^{2}+z^{2}\right)^{3 / 2}}-\frac{3 x^{2}}{\left(x^{2}+y^{2}+z^{2}\right)^{5 / 2}}\right\rfloor
\end{aligned}
$$

$$
\begin{gathered}
\frac{\partial u}{\partial x}=A\left\lfloor\frac{1}{\left(x^{2}+y^{2}+z^{2}\right)^{3 / 2}}-\frac{3 x^{2}}{\left(x^{2}+y^{2}+z^{2}\right)^{5 / 2}}\right\rfloor \\
\therefore \frac{\partial u}{\partial x}+\frac{\partial v}{\partial y}+\frac{\partial w}{\partial z}=A\left\lfloor\frac{3}{\left(x^{2}+y^{2}+z^{2}\right)^{3 / 2}}-\frac{3\left(x^{2}+y^{2}+z^{2}\right)}{\left(x^{2}+y^{2}+z^{2}\right)^{5 / 2}}\right\rfloor \\
=A\left\lfloor\frac{3}{\left(x^{2}+y^{2}+z^{2}\right)^{3 / 2}}-\frac{3}{\left(x^{2}+y^{2}+z^{2}\right)^{3 / 2}}\right\rfloor=0 \\
\therefore \frac{\partial u}{\partial x}+\frac{\partial v}{\partial y}+\frac{\partial w}{\partial z}=0
\end{gathered}
$$

Hence it is seen that these three velocities components satisfy the equation of continuity.

Now we try to check these velocity components when there will have four dimensions.

Let us we take these velocity components as follow,

$$
\begin{aligned}
& u(x, y, z, s)=\frac{A x}{\left(x^{2}+y^{2}+z^{2}+s^{2}\right)^{2}} \\
& v(x, y, z, s)=\frac{A y}{\left(x^{2}+y^{2}+z^{2}+s^{2}\right)^{2}} \\
& w(x, y, z, s)=\frac{A z}{\left(x^{2}+y^{2}+z^{2}+s^{2}\right)^{2}} \\
& r(x, y, z, s)=\frac{A s}{\left(x^{2}+y^{2}+z^{2}+s^{2}\right)^{2}}
\end{aligned}
$$

and

$\frac{\partial u}{\partial x}=\frac{\partial}{\partial x}\left(\frac{A x}{\left(x^{2}+y^{2}+z^{2}+s^{2}\right)^{2}}\right)$

$$
\begin{aligned}
& =\frac{\left(x^{2}+y^{2}+z^{2}+s^{2}\right)^{2} A \frac{\partial x}{\partial x}-A x \cdot 2\left(x^{2}+y^{2}+z^{2}+s^{2}\right) \cdot 2 x}{\left(x^{2}+y^{2}+z^{2}+s^{2}\right)^{4}} \\
& =\frac{A\left(x^{2}+y^{2}+z^{2}+s^{2}\right)^{2}-A 4 x^{2}\left(x^{2}+y^{2}+z^{2}+s^{2}\right)}{\left(x^{2}+y^{2}+z^{2}+s^{2}\right)^{4}}
\end{aligned}
$$

$\therefore \frac{\partial u}{\partial x}+\frac{\partial v}{\partial y}+\frac{\partial w}{\partial z}+\frac{\partial r}{\partial s}$

$$
\begin{aligned}
& =\frac{A \cdot 4\left(x^{2}+y^{2}+z^{2}+s^{2}\right)^{2}-A \cdot 4\left(x^{2}+y^{2}+z^{2}+s^{2}\right)^{2}}{\left(x^{2}+y^{2}+z^{2}+s^{2}\right)^{4}} \\
& =0
\end{aligned}
$$

Thus we have found that these components of velocity also satisfy these equation of continuity. 


\section{RESULTS AND DISCUSSION}

As it is seen in above research paper that a two dimension flow of any fluid which is incompressible satisfies the equation of

continuity. The components of flow has been taken as

$$
\begin{aligned}
& u(x, y)=\frac{A x}{\left(x^{2}+y^{2}\right)^{2 / 2}} \\
& v(x, y)=\frac{A y}{\left(x^{2}+y^{2}\right)^{2 / 2}}
\end{aligned}
$$

Similarly a three dimensional flow

$$
\begin{aligned}
& u(x, y, z)=\frac{A x}{\left(x^{2}+y^{2}+z^{2}\right)^{3 / 2}} \\
& v(x, y, z)=\frac{A y}{\left(x^{2}+y^{2}+z^{2}\right)^{3 / 2}} \\
& w(x, y, z)=\frac{A z}{\left(x^{2}+y^{2}+z^{2}\right)^{3 / 2}}
\end{aligned}
$$

Satisfies the equation of continuity. We have also check the such type of flow in four dimensions as,

$$
\begin{aligned}
& u(x, y, z, s)=\frac{A x}{\left(x^{2}+y^{2}+z^{2}+s^{2}\right)^{4 / 2}} \\
& v(x, y, z, s)=\frac{A y}{\left(x^{2}+y^{2}+z^{2}+s^{2}\right)^{4 / 2}} \\
& w(x, y, z, s)=\frac{A z}{\left(x^{2}+y^{2}+z^{2}+s^{2}\right)^{4 / 2}}
\end{aligned}
$$

and

$$
r(x, y, z, s)=\frac{A s}{\left(x^{2}+y^{2}+z^{2}+s^{2}\right)^{4 / 2}}
$$

These components of flow too satisfy the equation of continuity in incompressible flow.

From these observations we have got that we can generalize these components of flow upto n-dimension as follow

$$
\begin{aligned}
& u_{1}\left(x_{1}, x_{2}, \ldots \ldots, x_{n}\right)=\frac{A x_{1}}{\left(x_{1}^{2}+x_{2}^{2}+\ldots \ldots .+x_{n}^{2}\right)^{n / 2}} \\
& u_{2}\left(x_{1}, x_{2}, \ldots \ldots, x_{n}\right)=\frac{A x_{2}}{\left(x_{1}^{2}+x_{2}^{2}+\ldots \ldots .+x_{n}^{2}\right)^{n / 2}}
\end{aligned}
$$

$$
u_{n}\left(x_{1}, x_{2}, \ldots \ldots, x_{n}\right)=\frac{A x_{n}}{\left(x_{1}^{2}+x_{2}^{2}+\ldots \ldots .+x_{n}^{2}\right)^{n / 2}}
$$

If we consider the flow coordinate from the origin, then we can write these components as follow,

$$
\begin{aligned}
& u_{1}\left(x_{1}, x_{2}, \ldots \ldots, x_{n}\right)=\frac{A x_{1}}{\left(\sqrt{x_{1}^{2}+x_{2}^{2}+\ldots \ldots .+x_{n}^{2}}\right)^{n}}=\frac{A x_{1}}{D^{n}} \\
& u_{2}\left(x_{1}, x_{2}, \ldots \ldots, x_{n}\right)=\frac{A x_{2}}{D^{n}} \\
& u_{n}\left(x_{1}, x_{2}, \ldots \ldots, x_{n}\right)=\frac{A x_{n}}{D^{n}}
\end{aligned}
$$

Here A is any arbitrary constant and D is distance of the flow coordinate from the origin.

\section{GENERALIZATION}

In any incompressible n-dimensional flow the velocity component

$$
\begin{aligned}
& u_{1}\left(x_{1}, x_{2}, \ldots \ldots, x_{n}\right)=\frac{A x_{1}}{D^{n}} \\
& u_{2}\left(x_{1}, x_{2}, \ldots \ldots, x_{n}\right)=\frac{A x_{2}}{D^{n}}
\end{aligned}
$$

$$
u_{n}\left(x_{1}, x_{2}, \ldots \ldots, x_{n}\right)=\frac{A x_{n}}{D^{n}}
$$

will satisfy the equation of continuity.

\section{REFERENCES}

[1] Hydrodynamics, Fifteenth Edition 2005, KRISHNA Prakashan Media (P) Ltd. SHANTISWARUP.

[2] Fluid Dynamics with complete Hydrodynamics and Boundary Layer Theorem. Revised Edition S. CHAND \& COMPANY PVT. LTD. RAISINHANIA M.D. ISBN 81-219-0869-8. 
International Journal of Applied Engineering Research ISSN 0973-4562 Volume 16, Number 2 (2021) pp. 85-88 (C) Research India Publications. http://www.ripublication.com

[3] An Introduction to Fluid Dynamics, Cambridge Mathematical Library edition 2000. Batchelor G.K. CAMBRIDGE UNIVERSITY PRESS ISBN 0521663962

[4] Elements of Fluid Dynamics. Imperial College Press. Guido Buresti. ICP Fluid Mechanics, Vol. 3, ISBN13978-1-84816-888-6.

[5] Mechanical Sciences. Engineering Thermodynamics and Fluid Mechanics. Paul A.R., Mukherjee S., Roy P. Prentice Hall of India Private Limited. ISBN 81-2032727-6. 